\title{
Treatment of patients with glioma during the COVID-19 pandemic: what we learned and what we take home for the future
}

\author{
Federico Pessina, MD, ${ }^{1,5}$ Pierina Navarria, MD, ${ }^{2}$ Luisa Bellu, MD, ${ }^{2}$ Elena Clerici, MD, ${ }^{2}$ \\ Letterio Salvatore Politi, MD, ${ }^{3,5}$ Maria Pia Tropeano, MD, ${ }^{1}$ Matteo Simonelli, MD, ${ }^{4,5}$ \\ Maurizio Fornari, MD, ${ }^{1}$ and Marta Scorsetti, MD2,5
}

Departments of ${ }^{1}$ Neurosurgery, ${ }^{2}$ Radiotherapy and Radiosurgery, ${ }^{3}$ Neuroradiology, and ${ }^{4}$ Oncology and Hematology, Humanitas Clinical and Research Hospital-IRCCS, Rozzano; and ${ }^{5}$ Department of Biomedical Sciences, Humanitas University, Pieve Emanuele-Milan, Italy

OBJECTIVE Coronavirus disease 2019 (COVID-19) has changed the way in which cancer is treated. Patients with highgrade glioma (HGG) are believed to be in a vulnerable category. The aim of this study was to describe the experience of a hub cancer center and the measures that were put in place for treatment of patients with newly diagnosed and recurrent glioma.

METHODS To prevent in-hospital contagion and preserve the safety of health professionals and patients, specific protocols and strict regulations were introduced. Physical distancing, use of surgical masks, and diligent hand hygiene were adopted. Each case was discussed in a multidisciplinary board meeting before treatment. All patient candidates for surgical procedures were tested for SARS-CoV-2 with a nasopharyngeal swab and a chest CT scan. Indications for surgery were the radiological suspicion of HGG in patients with a good performance status and/or the rapid and progressive occurrence of neurological deficits. Adjuvant treatments were performed only in cases of HGG. This therapy consisted of conventional fractional radiotherapy (RT; $60 \mathrm{~Gy} / 30$ fractions) with concomitant and adjuvant temozolomide chemotherapy (TMZCHT) in younger patients; in elderly patients, a short course of RT was employed (40.5 Gy/15 fractions). For recurrent HGG, treatments were assessed after a careful evaluation of the patient's general condition, neurological status, and risk of early impairment in neurological status if not treated. During simulation CT for the RT plan, each patient underwent a chest CT study. In cases in which an imaging study was suspicious for COVID-19 pneumonia, the patient was immediately isolated and rapidly underwent nasopharyngeal swab testing.

RESULTS Between March 1 and April 30, 2020, 23 HGGs were treated, and these cases are included in the present evaluation. Fifteen patients harboring newly diagnosed glioblastoma (GBM) underwent resection followed by a regimen of chemotherapy and RT, and 3 patients with newly diagnosed anaplastic oligodendroglioma underwent surgery followed by adjuvant RT. Five patients were treated for recurrent GBM, and they received surgery plus adjuvant RT. One patient in whom the simulation CT study was suspicious for COVID pneumonia was tested with a nasopharyngeal swab, which proved positive for SARS-CoV-2 infection. No patients contracted COVID-19 during hospitalization for surgery or during RT treatment. Corticosteroid therapy was administered to all patients beginning on the 1st day of RT.

CONCLUSIONS The authors' experience during the COVID-19 pandemic showed that patients with HGG can be treated in the most effective manner without a compromise in safety. Careful selection criteria and a multidisciplinary evaluation are pivotal to assessing the optimal therapeutic strategy.

https://thejns.org/doi/abs/10.3171/2020.9.FOCUS20704

KEYWORDS high-grade glioma; COVID-19 pandemic; surgery; radiation therapy; chemotherapy

$\mathrm{S}$ INCE December 2019, when severe acute respiratory syndrome coronavirus 2 (SARS-CoV-2) was first identified in Wuhan, Hubei, China, the epidemic has dramatically evolved into a worldwide pandemic ex- erting a disruptive health, social, and economic impact. ${ }^{1-4}$ Italy was one of the countries most affected by the pandemic, above all in the northern regions. ${ }^{5-8}$ In the midst of this pandemic scenario, the Italian cancer community

ABBREVIATIONS GBM = glioblastoma; HGG = high-grade glioma; KPS = Karnofsky Performance Status; PPE = personal protective equipment; RT = radiotherapy; 
found itself having to face and manage particularly difficult situations. It would seem that cancer patients may be more susceptible to infections because of their immunosuppressive status. ${ }^{9-11}$ This is particularly the case in patients with a diagnosis of glioma, the most common primary brain tumor. Patients with glioma are considered one of the most fragile and vulnerable cancer populations due to several factors: 1) a tendency toward old age along with multiple age-related comorbidities; 2) increased risk of thromboembolic events related to the patient's loss of autonomy, the tumor, and/or chemotherapy treatments; and 3) the large use of steroid medications, which produce a further increase in the patient's immunosuppression status and, consequently, a greater susceptibility to infections. ${ }^{12-14}$ Nevertheless, a delay in oncological care could, in the same way, heavily affect the patient's survival. Indeed, malignant primary brain tumor, if not promptly treated, may involve a higher risk of impairment in neurological status and the patient's quality of life, and therefore survival.

In the most recent months, there have been several treatment recommendations published by national and international associations as well as federal, state, and local governing institutions. ${ }^{15-19}$ These suggestions are related to patient characteristics (age, performance status, and neurological status), glioma features (histological characteristics and molecular profile), and tumor primary or recurrent status. In patients in whom MRI raises suspicion for highgrade glioma (HGG), a maximal safe resection should be performed to make a definitive diagnosis and create a tailored treatment plan, especially in younger adults with a good performance status. Adjuvant treatments consisting of conventional fractionated radiotherapy (RT), more or less with concomitant and maintenance temozolomide chemotherapy (TMZCHT), are indicated. In elderly and frail patients, surgical biopsy or resection may need to be abrogated, and further treatment strategies may have to be based on radiological findings alone. Among the latter, as proposed by randomized trials, a shorter RT course should be scheduled, and, in this particular pandemic situation, even a 1-week course of RT may be proposed. For recurrent gliomas, for which a standard of care has not yet been well defined, clinical trials constitute an important patient option, but unfortunately, protocols may require modifications to minimize exposure to SARS-CoV-2. ${ }^{2-14}$

At the time of writing, in Italy we have just overcome the maximum crisis, but all hospitals in the northern regions of Italy faced an emergency that was well off the charts, causing drastic changes in all processes of care. Comprehensive data on glioma patients who were affected by SARS-CoV-2 infection are still not available. Given the lack of such data, in this paper we would like to describe our experiences in a hub cancer center situated in the "red zone" of Italy and the measures that we put in place for the management of glioma cases.

\section{Methods}

\section{General Measures Undertaken}

To prevent in-hospital SARS-CoV-2 contagion and preserve the safety of health professionals and patients, we quickly introduced the following specific protocols and strict regulations:

- The entire hospital was divided into two different areas: a "contaminated" zone for patients with suspected or confirmed infections, and a "clean" area only accessible by outpatients and health workers. Crossing between these two zones was strictly forbidden in order to reduce contamination.

- We imposed physical distancing and a limit on all unnecessary interactions between patients and medical staff during the clinical interview and in every other unnecessary situation. To facilitate this directive, we limited access to the hospital for all caregivers, except in cases of nonautonomous patients.

- We activated triage points at every entrance to the hospital and departments, where we measured body temperature using infrared thermometers to maintain some distance and supplied surgical masks for everyone. Before access to the hospital and/or before examination, every patient was interviewed concerning the presence of any suspected symptoms.

- From the first weeks of the virus spread in Italy, we began to routinely use surgical masks at every facility and also introduced the use of fitted respiratory masks, such as FFP2, for health workers in every situation in which there was close patient contact, in accordance with World Health Organization indications. ${ }^{15-19}$

- At the same time, we implemented diligent hand hygiene by educating the medical staff as well as patients in the use of hydroalcoholic solutions. Medical staff, in particular, practiced hand hygiene before and after any contact with patients, before meals, and before touching medical devices.

- To define the optimal therapeutic strategy, each case was and is discussed in a multidisciplinary board meeting before treatments.

\section{Surgical Measures Undertaken}

All patient candidates for surgical procedures were tested with a nasopharyngeal swab to detect the presence of SARS-CoV-2 and also a chest CT scan to exclude the presence of COVID-19 pneumonia. The indication for hospitalization was confirmed only after these tests, performed in a "gray" area on an outpatient basis, proved negative. In cases of elective surgery in which nasopharyngeal swab testing or CT provided positive findings, the patient followed the path of quarantine or hospital stay according to clinical status. In cases of emergency procedures, in which it was impossible to perform any type of test and impossible to delay surgery, we evaluated whether to perform the procedure using advanced personal protective equipment (PPE) in dedicated operating rooms or to postpone it. Indications for surgery in this difficult scenario were substantially the radiological suspicion for high-grade malignancy in patients with a good performance status (Karnofsky Performance Status [KPS] scores 80-100) and/ or the rapid and progressive occurrence of neurological deficits. Exclusion criteria included a multicentric tumor or a recurrent HGG located in an eloquent area. Surgery was performed in all patients with the aim of maximum 
removal of the tumor mass according to functional boundaries, which was undertaken with the aid of imaging and brain mapping techniques. Although SARS-CoV-2 transmission during an awake craniotomy has never been reported, theoretically there is a high risk of viral transmission while the patient is breathing and speaking. It may be prudent to use nonawake strategies for eloquent area mapping, while in cases of large masses involving speech areas, the need of biopsy alone should be discussed. Postoperatively, both in the intensive care unit and neurosurgical ward, the presence of relatives during the patient's stay was not allowed, and every communication regarding the patient's condition and outcome was conducted by phone or teleconferencing session.

\section{Adjuvant Treatments}

As indicated by expert consensus recommendations, adjuvant therapy was delayed for asymptomatic patients who harbored a low-grade astrocytoma diagnosed with a $1 \mathrm{p} / 19 \mathrm{q}$ codeletion and an isocitrate dehydrogenase (IDH) mutation because of these tumors' alleged slow growth rate. ${ }^{12-14}$ In contrast, for patients with HGG, for whom the risk of delaying adjuvant treatment could exceed the risk of SARS-CoV-2 contagion, adjuvant treatments were evaluated. Younger patients with a newly diagnosed HGG received RT (60 Gy/30 fractions) over a 6-week period with concomitant and adjuvant TMZCHT. In elderly patients, a short course of RT (40.5 Gy/15 fractions) was administered over 3 weeks, more or less with concomitant and adjuvant TMZCHT, depending on the patient's KPS score and $\mathrm{O}^{6}$-methylguanine-DNA-methyltransferase (MGMT) promoter methylation status. For cases of recurrent HGGs, treatments were assessed after careful evaluation of the patient's general condition, neurological status, and risk for an early impairment in neurological status if not treated. Corticosteroid medication was administered at lower doses from the 1st day of RT.

To rapidly identify whether patients were infected with SARS-CoV-2, even if asymptomatic, we introduced a new strategy in our radiotherapy department: each patient who underwent standard CT simulation for subsequent RT also underwent a chest CT scan. In cases in which a CT study was suspicious for COVID-19 pneumonia, an expert radiologist evaluated the CT scans; if the radiologist confirmed the radiological suspicion, the patient was immediately isolated and rapidly underwent nasopharyngeal swab testing. The CT-simulation room was thoroughly disinfected before receiving a new patient.

\section{Results}

Between March 1 and April 30, 2020, among all cases of brain tumors that were evaluated at our institution, 23 HGGs were treated; these cases are included in the present evaluation. Fifteen patients harboring these tumors were men and 8 were female; the median patient age was 53 years (range $42-80$ years). Twenty patients (87\%) had KPS scores of 90-100, while only 3 patients (all with recurrent tumors) had a KPS score of 80. Eight patients in the cohort displayed neurological deficits: 2 patients with hemianopia, 4 patients with language disturbances, and 2 patients (recurrent tumors) with mild motor impairments. No further neurological deficits were recorded after surgery, and among patients with preoperative deficits the neurological findings remain stable. Fifteen patients harboring newly diagnosed glioblastoma (GBM) underwent resection followed by RT plus concomitant and adjuvant TMZCHT. Three patients with newly diagnosed anaplastic oligodendroglioma underwent surgery followed by adjuvant RT. Thirteen patients received conventional fractionated RT consisting of $60 \mathrm{~Gy}$ in 30 daily fractions over 6 weeks, while 10 elderly patients received 40.5 Gy in 15 fractions over 3 weeks. Five patients were treated for recurrent GBM; they underwent surgery plus adjuvant RT (25 Gy/5 fractions over 1 week). One patient from southern Italy with recurrent GBM delayed treatment due to the pandemic. When hospitalization for surgery could be planned for this patient, a wide disease progression was recorded and an impairment in neurological status was observed. At this time only best supported care was indicated. During simulation for RT planning, radiological imaging revealed suspected COVID pneumonia in an asymptomatic patient, and a subsequent nasopharyngeal swab tested positively for SARS-CoV-2 infection. Adjuvant treatment in that patient had to be postponed until further swab testing proved negative, after which the patient completed RT without side effects. No patients contracted COVID-19 disease during hospitalization for surgery or during RT, allowing the patients to undergo the entire therapy without complications. Corticosteroid therapy was administered to all patients from the 1st day of RT; no dosage increase was needed.

\section{Discussion}

Italy was one of the countries most seriously affected by the COVID-19 pandemic, particularly in the northern regions (Lombardy, Veneto, and Emilia-Romagna). There is concern that cancer patients and cancer survivors are more likely to become infected with the novel coronavirus and are more likely to die from complications of COVID-19. ${ }^{6}$ In this scenario, avoidance of exposure and prevention of infection are crucial issues. Challenging topics include determining which patients require early treatment, how treatment can be delayed, and how we can offer optimal care while minimizing the potential risk of infections. The first point is patient selection. The neurooncological international guidelines helped and supported us in making choices. ${ }^{12-14}$ In patients with presumed benign tumors and tolerable symptoms, surgery, radiosurgery, and any adjuvant RT should be deferred. In patients with alleged low-grade gliomas with a presumed slow growth rate, an observational approach rather than active treatment may be optimal. Diagnostic surgery and adjuvant therapy can be safely delayed, especially if the patient presents with only limited and stable neurological symptoms, and further tumor growth will not preclude complete resection. Patients in whom neuroimaging features are suggestive of HGG comprise a subset of patients in whom a delay of oncological care may pose a higher risk of impairment in neurological status, quality of life, and therefore survival. Therefore, in a pandemic scenario, pa- 
tients with HGG still require treatment that is multimodal and impossible to postpone. In these patients, integration of the molecular features of the tumor, the extent of resection, and the patient's age and performance status are critical to predict a prognosis as well as the potential benefit of added therapies.

In this context, at the end of the so-called phase 1 of the pandemic, our neuro-oncological board decided to retrospectively analyze the outcome of HGG patients treated at our institution. The primary aim was to evaluate the appropriateness of the prevention measures that were put in place to ensure the safety of patients and the healthcare staff, the criteria followed to treat patients during such a dramatic situation, and any changes in treatment choices induced by the presence of the pandemic. We focused our attention on patients with HGG, because these tumors represent a subset in which there is faster tumor growth and a histomolecular diagnosis is crucial to address postoperative cures and a cytoreduction impact on survival,${ }^{14}$ and because treatment of these tumors is necessarily multimodal and difficult to delay. In this context, patients with HGG are the most fragile and at risk for inappropriate criteria and timing of treatment. We therefore evaluated every HGG case in a multidisciplinary board meeting to share common criteria regarding the timing and modalities of treatments. In accordance with our experience, patients without severe comorbidities in whom there was radiological suspicion of HGG underwent surgical treatment followed by various chemoradiation schedules based on patient age and tumor molecular assessment. We agreed to avoid surgery in cases of HGG recurrence located in eloquent areas due to the higher risk of complications and longer hospitalization time. As of the end of COVID-19 phase 1, 23 patients with HGG had been treated at our institution, one-third fewer than during the same period of the previous year. This fact was related to logistical problems, difficulty in traveling between different regions of the same country, and a non-negligible patient fear about transfers to health centers. It could seem questionable whether this kind of approach can be considered right or advisable should a new pandemic arise, but some considerations deserve to be made. First, the treatments were safe. In fact, notwithstanding the frailty of these cancer patients, surgical treatment followed by RT, chemotherapy, and corticosteroid medications were instituted and only one of these patients became COVID-19 positive. That one patient was completely asymptomatic, and the infection was detected by chest CT during brain CT simulation, a protocol that our RT department established to increase safety and accuracy in this cohort of frail patients. Second, the choice to assign RT schedules according to each patient's age and general condition allowed us to treat with efficacy and without complications a large spectrum of HGG patients during this dramatic time period. Our selection strategy and approach, always discussed by a multidisciplinary board, permitted us to provide multimodal treatment in accordance with patient and/or disease characteristics, without having to shift toward a different therapeutic option. It is clear that the high standards of the institution (indication for hospitalization was confirmed only if both the chest CT study and naso- pharyngeal test, performed in a "gray" area on an outpatient basis, produced negative findings of COVID-19), the availability of advanced PPE, the modification of surgical approaches (for example, the choice of only asleep surgeries), the chest CT scan performed during RT simulation, and the various schedules of RT that could be selected were fundamental for better management of HGG cases.

It is not possible to establish a "crucial" factor among the preceding considerations in successfully preventing COVID-19 infection during cures of this cohort of complex patients. Probably, the correct and constant interactions among COVID-19 prevention, the diagnostic strategies, and the standardization of HGG patient selection are pivotal. On the other side, it is not to be underestimated that the limited number of patients treated during the pandemic period could play a role in infection containment in our cohort. The experience gained in this context can be extremely useful when faced with a new pandemic emergency, when neuro-oncological patients must be treated, without delay, in reference structures with expertise and a high standard of security.

\section{Conclusions}

Our experience during the COVID-19 pandemic showed that it is possible to preserve a high quality of cures, even in this difficult scenario, and that patients with primary brain tumors can be treated in the most effective way without compromising their safety. Careful selection criteria and a multidisciplinary evaluation are pivotal to assessing the optimal therapeutic strategy.

\section{References}

1. Zhou F, Yu T, Du R, et al. Clinical course and risk factors for mortality of adult inpatients with COVID-19 in Wuhan, China: a retrospective cohort study. Lancet. 2020;395(10229): 1054-1062.

2. Hoehl S, Rabenau H, Berger A, et al. Evidence of SARSCoV-2 infection in returning travelers from Wuhan, China. $N$ Engl J Med. 2020;382(13):1278-1280.

3. Rothe C, Schunk M, Sothmann P, et al. Transmission of 2019-nCoV infection from an asymptomatic contact in Germany. N Engl J Med. 2020;382(10):970-971.

4. Huang C, Wang Y, Li X, et al. Clinical features of patients infected with 2019 novel coronavirus in Wuhan, China. Lancet. 2020;395(10223):497-506.

5. Remuzzi A, Remuzzi G. COVID-19 and Italy: what next? Lancet. 2020;395(10231):1225-1228.

6. Vergano M, Bertolini G, Giannini A, et al. Clinical ethics recommendations for the allocation of intensive care treatments in exceptional, resource-limited circumstances: the Italian perspective during the COVID-19 epidemic. Crit Care. 2020;24(1):165.

7. Implementing provisions of decree-law 23 February 2020, n. 6 , concerning urgent measures regarding the containment and management of the epidemiological emergency COVID-19, and subsequent updates and changes. Official Gazette of the Italian Republic. Article in Italian. Accessed October 15, 2020. https://www.gazzettaufficiale.it/eli/ id/2020/03/11/20A01605/SG

8. Regional Council decree N XI/2906 concerning new additional determinations concerning the epidemiological COVID-19 public health emergency. Article in Italian. Accessed October 15, 2020. https://www.regione.lombardia.it/ 
wps/wcm/connect/5e0deec4-caca-409c-825b-25f781d8756c/ DGRp2906p8pmarzop2020.pdf?

9. Kessler RA, Zimering J, Gilligan J, et al. Neurosurgical management of brain and spine tumors in the COVID-19 era: an institutional experience from the epicenter of the pandemic. $J$ Neurooncol. 2020;148(2):211-219.

10. Wang H, Zhang L. Risk of COVID-19 for patients with cancer. Lancet Oncol. 2020;21(4):e181.

11. Dai M, Liu D, Liu M, et al. Patients with cancer appear more vulnerable to SARS-CoV-2: a multicenter study during the COVID-19 outbreak. Cancer Discov. 2020;10(6):783-791.

12. Mohile NA, Blakeley JO, Gatson NTN, et al. Urgent considerations for the neuro-oncologic treatment of patients with gliomas during the COVID-19 pandemic. Neuro Oncol. 2020;22(7):912-917.

13. Bernhardt D, Wick W, Weiss SE, et al. Neuro-oncology management during the COVID-19 pandemic with a focus on WHO grade III and IV gliomas. Neuro Oncol. 2020;22(7): 928-935.

14. Weller M, Preusser M. How we treat patients with brain tumour during the COVID-19 pandemic. ESMO Open. 2020; 4(Suppl 2):e000789.

15. Zhang $\mathrm{H}-\mathrm{W}, \mathrm{Yu} \mathrm{J}, \mathrm{Xu} \mathrm{H}-\mathrm{J}$, et al. Corona virus international public health emergencies: implications for radiology management. Acad Radiol. 2020;27(4):463-467.

16. Feng S, Shen C, Xia N, et al. Rational use of face masks in the COVID-19 pandemic. Lancet Respir Med. 2020;8(5): 434-436.

17. Alhazzani W, Møller MH, Arabi YM, et al. Surviving Sepsis Campaign: guidelines on the management of critically ill adults with Coronavirus Disease 2019 (COVID-19). Intensive Care Med. 2020;46(5):854-887.
18. Chen W, Su X-Y, Wang VJ, et al. Novel coronavirus international public health emergency: guidance on radiation oncology facility operation. Adv Radiat Oncol. 2020;5(4): $560-566$.

19. World Health Organization. Infection prevention and control during health care when novel coronavirus (nCoV) infection is suspected: interim guidance. March 19, 2020. Accessed October 15, 2020. https://www.who.int/publications/i/ item/10665-331495

\section{Disclosures}

The authors report no conflict of interest concerning the materials or methods used in this study or the findings specified in this paper.

\section{Author Contributions}

Conception and design: Pessina, Navarria. Acquisition of data: Pessina, Navarria, Bellu, Tropeano. Analysis and interpretation of data: Pessina, Navarria. Drafting the article: Pessina, Navarria, Bellu. Critically revising the article: Pessina, Navarria, Clerici, Politi, Simonelli, Fornari. Reviewed submitted version of manuscript: Pessina, Navarria, Simonelli, Fornari, Scorsetti. Approved the final version of the manuscript on behalf of all authors: Pessina. Study supervision: Pessina.

\section{Correspondence}

Federico Pessina: Humanitas Cancer Center and Research Hospital, Rozzano, Italy. federico.pessina@hunimed.eu. 